\title{
Género y Feminización de los Espacios Extensionistas Latinoamericanos
}

\section{Genderand Feminization of Latin Amrican Extensionist Spaces}

Sylvia María Valenzuela Tovar ${ }^{1}$

Red Nacional de Extensión Universitaria ASCUN

https://orcid.org/0000-0002-9416-7631

sylvialaboral@gmail.com

Fecha de recibido: 10/04/2021

\section{Resumen}

Conscientes de la importancia e impacto de la función universitaria de extensión o proyección social en el contexto colombiano y latinoamericano como dialogo de saberes y herramienta de transformación y desarrollo, durante los últimos años, diferentes instituciones de la región, han venido realizando el seguimiento al rol femenino en el ejercicio de dicha función.

El presente documento contiene entonces una síntesis de lo encontrado al respecto durante el último quinquenio, permitiendo a los lectores conocer el cierre de brechas de género en la dirección, planeación y gestión de la extensión universitaria en las muchas instituciones de educación superior latinoamericanas tanto públicas como privadas. Lo anterior a partir de una revisión documental y una serie de entrevistas realizadas a los responsables de la función universitaria en 20 países de la región y alrededor de 180 instituciones.
Fecha de dictaminado: 05/05/2021

El texto habla de mujeres directoras, jefes y gestoras de la extensión universitaria o proyección social y propone una reflexión en torno a los procesos de feminización, a los sujetos involucrados en la función universitaria, así como al tipo de participación de hombres y mujeres en los equipos extensionistas.

\section{Palabras claves}

Hombre, mujer, género, extensión universitaria, feminización, Latinoamérica.

\section{Summary}

Aware of the importance and impact of the university function of extension or social projection in the Colombian and Latin American context as a tool for transformation and development, in recent years Colombia has been monitoring the role of women in the exercise of said university function.

1 Licenciada en Educación, Especialista en Docencia Universitaria. Miembro de la Unión Latinoamericana de Extensión Universitaria-ULEU y enlace oficial de dicha red para Colombia. Integrante de la Red de docentes de América Latina y del caribe (RedDOLAC). Miembro del Comité Nacional de Extensión Universitaria ASCUN Colombia. Profesora conferencista invitada y asesora de diferentes Universidades Colombianas y Latinoamericanas. Miembro del grupo CLACSO "Extensión Critica: teorías y prácticas en América Latina y Caribe". Asesora de cooperación y conferencista internacional en temas de Extensión, Proyección Social, Vinculación e Infancia. Contacto: sylvialaboral@gmail.com https://orcid.org/0000-0002-9416-7631 
This document then contains a synthesis of what was found in this regard during the last five years, allowing readers to know the closing of gender gaps in the direction, planning and management of university extension in the multiple Latin American higher education institutions, both public and private... This is based on a documentary review and a series of interviews carried out with those responsible for the university function in 20 countries of the region and around 180 institutions.

Thus, the text speaks of women directors, heads and managers of the university extension or social projection and proposes a reflection on the feminization processes, the subjects involved in the university function, as well as the type of participation in the extension teams.

\section{Keywords}

Man, woman, gender, universitary extension, feminization, Latin-American.

\section{Introducción}

El reconocimiento del ejercicio de la Extensión Universitaria, Vinculación o Proyección Social a nivel latinoamericano y caribeño durante los últimos años, ha estado acompañado de una fuerte presencia femenina en los cargos jerárquicos-directivos y la constitución de los equipos gestores de la misma función, alimentando el debate y la reflexión en torno a la feminización de los espacios extensitas. Para entender dicha situación es necesario partir de un breve recorrido a nivel mundial de lo que ha sido la presencia de la mujer en la educación superior y por ende, en la extensión universitaria o vinculación, para continuar con una pequeña reflexión en cuanto al ser y quehacer mismo de esta función sustantiva, lo que permite dar paso a la presentación y resultados del estudio que ha dado origen a este texto.

La extensión universitaria, a lo largo de la historia, ha sido entendida y gestionada como una serie de oportunidades de acceso a la educación superior para las poblaciones excluidas, el conjunto de acciones altruistas de la comunidad universitaria implementadas mediante pasantías sociales a favor de los territorios marginados, o la suma de acciones divulgativas que buscan acercar a la población a los avances tecnológicos y las expresiones culturales creadas por las mismas instituciones educativas.
Pero también ha sido vista, como el conjunto de acciones de carácter concientizador que procura generar mecanismos de análisis y reflexión a partir del vínculo universidad-entorno con un componente muy cuestionado que no tiene cabida en la concepción dialógico-crítica de la misma como lo es, la búsqueda de recursos económicos - por esta vía - para la misma universidad. Sin embargo, en otros momentos de la historia, la extensión universitaria ha servido de carta de presentación de las instituciones como medio que les permite proyectarse en los territorios, también ha sido entendida y gestionada a manera de frontera o encuentro entre la institución y la sociedad misma, como una membrana permeable que le permite a las universidades un determinado intercambio con su entorno inmediato, en otras palabras es una estrategia bidireccional que facilita el llevar, dejar y enseñar pero solo hacia fuera de la institución, o finalmente ha sido concebida como un dialogo de saberes crítico, horizontal y de doble vía que facilita la integración e interacción para la construcción de una mejor sociedad. Sin embargo, independientemente de cual sea la concepción de esta función universitaria, la creciente presencia femenina en estos escenarios ha influido en la actual forma de gestionarla (11 tipologías).

Esta feminización de los espacios y escenarios extensionistas, ha venido en aumento durante los últimos años a nivel latinoamericano y caribeño, sin embargo, en algunos países y universidades de la región, persisten los desequilibrios desde el momento mismo de designar los cargos jerárquicos-administrativos o de constituir los equipos gestores extensionistas. Esto se convierte en un reto institucional y un desafío regional.

\section{Antecedente}

\section{Mujeres, historia y extensión universitaria}

La presencia femenina en los claustros universitarios y en general en la educación superior mundial, fue muy pobre hasta hace unas pocas décadas, pues la prevalencia de la masculinización de las actividades académicas, se da como consecuencia del sistema de patriarcal que generó en su momento, una serie de barreras socioculturales visibles, en especial durante la Edad Media; período en el que los claustros religiosos eran los únicos que permitían a las mujeres acceder a dichos procesos educativos. Lo anterior fue replicado en los diferentes países europeos y americanos con excepción de Italia hasta el siglo XIX, o incluso, en 
algunos otros pocos lugares hasta el siglo XX. Ello contrasta con la lucha durante siglos del género femenino por el derecho y acceso a la educación y que tan solo a partir de la segunda mitad del siglo XIX comenzó a hacerse realidad en países como Suiza (1860), Alemania (1900) Francia (1880), Gran Bretaña (1870) y Cambridge (1947). Previo a esto, existen antecedentes como los de la abadesa Bettisia Gozzadini, en la Universidad de Bolonia quien ejerció como docente universitaria luego de graduarse de abogada y de la que cuenta la historia que daba su clase con un velo, "para que su belleza no interfiriera en clase” (Matteucci, F, 2014).

Según Alicia Palermo (2006), durante la edad media, algunas mujeres que lograron acceder a los claustros universitarios y obtener sus títulos académicos como profesionales; años más tarde lograron desempeñarse como docentes en los mismos claustros. Entretanto, en países como Alemania, Francia e Inglaterra, la oportunidad para que las mujeres desempeñaran dichos cargos (docentes universitarias o directoras) estuvo dada en un primer momento, a razón de algunas cátedras, tutorías y consejerías impartidas desde los colegios privados para señoritas acaudaladas resaltando entre estas primeras mujeres docentes a Sophia Jex Blake fundadora, en 1892, de la Escuela de Medicina para mujeres, Magdalena Canedi(Universidad de Bologna, Italia), Lucía de Medrano (Universidad de Salamanca), María Pellegrina (Universidad de Pavía) y Anna María Van Shurman (Universidad de Utrech, Holanda). Todas ellas, mujeres docentes universitarias destacadas y pioneras en la historia, que dieron grandes pasos para el cierre de brechas de género en la educación superior.

A partir de la década de los 90, las mujeres comienzan a organizarse y reflexionar sobre las desigualdades a las que se enfrentaban (entre ellas los procesos universitarios y laborales jerárquicos o directivos). Pero no es sino hasta el año 2005 cuando el Instituto Internacional para la Educación Superior en América Latina y el Caribe IESALC, apoyado por la UNESCO, analiza los factores sociales, educativos y económicos de las matriculas de educación superior, enfatizando en el aspecto de género en las universidades. Fruto de ello, señala que:

"...en lo que respecta a la Educación Superior se observa como uno de los cambios más significativos de las últimas décadas, el crecimiento constante-cuando no mayoritario- de la matrícula y egresos femenina, cuestión que revierte un proceso de siglos, en el que predominaban los hombres en las aulas y laboratorios de las universidades latinoamericanas. Este proceso que luce irreversible es parte de un cambio epocal y está teniendo fuertes impactos sociales y tendrá una gran incidencia en la transformación de instituciones y centros de investigación, así como de las sociedades latinoamericanas en general (...) Dicho proceso ha sido denominado como Feminización de la Educación Superior" (Agostini, 2017)

Lo anterior concuerda con el hecho de que las mujeres, hasta hace tan solo un siglo, vienen alcanzando gradualmente una serie de logros entre los que figuran su derecho al voto, el acceso a la educación y a los centros de decisión política, su vinculación al mercado laboral púbico y privado, la visibilización de su gestión e impacto en la transformación de las sociedades, el ejercicio de roles jerárquicos y directivos laborales y por ende, entre ellos, la dirección de la función de Extensión Universitaria en muchas instituciones y países latinoamericanos y caribeños.

En 1991 FLACSO como Facultad Latinoamericana de Ciencias Sociales y organismo internacional de educación autónomo para América Latina y el Caribe, dedicado a la investigación, docencia y difusión de las ciencias sociales, ha manifestado mediante su informe titulado Mujeres latinoamericanas en cifras, que en los diferentes países de la región:

"La docencia es una profesión mayoritariamente femenina, excepto en las escuelas técnicas y en la universidad. Ellas representan la casi totalidad de los docentes en el nivel preescolar, tres cuartos en la escuela primaria, la mitad en la enseñanza secundaria y una minoría en la educación superior" (FLACSO, 1991).

Ahora bien, las cifras más recientes en los países del MERCOSUR, presentan un panorama alentador y cambiante. Casos como el de Uruguay, donde de acuerdo a Papadópulos y Radakovich (2003), el género femenino ocupa un lugar relevante en el porcentaje de la matrícula universitaria el que varía entre dos y tres cuartos de la misma, hace alusión a nuevos paradigmas y posibilidades, o casos como el de Argentina que pasó en 1970 de reportar un $36 \%$ de mujeres con estudios de Educación Superior completos a un 60\% en el 2001, a pesar de ello, los estudio muestran que en Brasil la diferencia en número persiste y persisten las brechas laborales de género . 
En el caso de Colombia, Paulina Beregoff (ucraniana) figura como la primera mujer graduada de una universidad en el territorio nacional, que para este caso fue la Universidad de Cartagena en 1925 y Mariana Arango Trujillo la primera colombiana en obtener un título profesional (Odontología en la Universidad de Antioquia, en 1937), no obstante, fue solo hasta 1933 que el gobierno mediante el Decreto 227, permitió la entrega del título de bachiller para las mujeres y en consecuencia la posibilidad de que éstas ingresaran a la Educación superior. Ahora, en cuanto a cargos de dirección y administrativos jerárquicos de las Instituciones de educación superior a nivel latinoamericano, un informe reciente de la UNESCO ha revelado que "solo un 18\% de universidades públicas latinoamericanas cuenten con mujeres en calidad de rectoras" (UNESCO, 2021), lo que concuerda con el caso colombiano en donde fue necesario que pasaran 42 rectores y 150 años desde la creación de la Universidad Nacional de Colombia, para que llegara en 2018 a la rectoría de ésta, catalogada como la mejor universidad del país, una mujer: Dolly Montoya Castaño. Sin embargo, como dirían Corzo y Galeano en su texto "Las mujeres ante la educación superior en Colombia”.

"Desde entonces, las mujeres han ido conquistando derechos a nivel global, y en el contexto colombiano los avances, aunque lentos, también se han ido incorporando a lo largo del siglo XX y de las dos primeras décadas del XXI." (Corzo Morales, M., \& Galeano Camacho, E. 2020).

Como puede observarse en los últimos 25 años, la presencia de la mujer ha cambiado notablemente en el ámbito universitario. Gran parte de los países latinos y europeos, han trabajado por superar las marcadas desigualdades a fin de alcanzar una casi equidad femenina que disminuya la brecha de género. Parafraseando a Maria Valpuesta (2012), la mujer se ha incorporado al mundo de lo público trascendiendo con creces las expectativas generadas y soportando en gran medida, la atención a los más necesitados sin ningún tipo de retribución. La mujer lo ha hecho, entonces, de manera cada vez mayor y visible a partir del siglo $\mathrm{XXI}$ a la educación superior y por ende a sus procesos sociales y extensionistas. Y qué mejor ejemplo de esta inclusión y presencia femenina en las universidades latinoamericanas que el informe del Observatorio de Derechos Humanos de la Universidad Nacional de Córdoba presentado en 2017, que evidencia:

"En el año 2006 la planta Nodocente de la Universidad estaba constituida por $41 \%$ de mujeres y un $59 \%$ de varones. 9 años después, en 2015, las mujeres representan el 60\%; es decir, que hubo un incremento de casi el $20 \%$ lo que refleja el fuerte proceso de feminización de este claustro universitario" (Observatorio de DD. HH. De la UNC, 2017)

Por su parte la Universidad Nacional de La Pampa también ha venido reflexionado al respecto, y en consecuencia ha afirmado que:

En los últimos años, el incremento de las iniciativas dirigidas y coordinadas por mujeres se han acentuado y superan al $75 \%$. Ello tuvo una marcada incidencia en la conformación de los equipos y las problemáticas planteadas (...) Este proceso de feminización de la matrícula universitaria, es decir, mayor número de mujeres como personas inscriptas, también se refleja en la extensión como función. Los datos señalan que son las mujeres quienes tienen mayor presencia en la dirección y coordinación de propuestas de extensión de las últimas cuatro convocatorias (correspondientes a los años 2016, 2017, 2018 y 2019). En la del año 2016 el $70 \%$ de los programas, proyectos y acciones fueron dirigidos por mujeres (docentes, no docentes y estudiantes), en 2017 el 73\%, en 2018 el 83\% y en 2019 alcanza el $78 \%$. En cuanto a la conformación de los equipos extensionistas, el número de participantes mujeres también es más elevado ya que en el último período alcanzó un 65\% (UNLPam, 2020).

De igual manera, pero mucho más al norte del continente americano, es posible encontrar otras investigaciones en las que se evidencia la feminización de la extensión, vinculación o proyección social. La universidad de Costa Rica, por ejemplo, analizó en el periodo del 2002 al 2017 el comportamiento de participación de mujeres tanto profesionales como estudiantes en: liderazgo, dirección y gestión de proyectos de extensión, hallando que:

\footnotetext{
"Las mujeres representan el 55\% de la población total de extensionistas, (...) Con respecto a los roles de jerarquía, se observa para el año 2016 que pasa a ser un 55\% a cargo de mujeres y un $45 \%$ a cargo de hombres (...) el $42 \%$ de las mujeres participan en proyecto integrados frente a un 35\% de hombres que también participan, y un 13\% de mujeres que trabajan directamente con proyectos de extensión frente a un 10\% de hombres que igualmente lo hacen" (Rojas Herrera Silvia en: Lovo S.M; Ramos M y Valenzuela S.M., 2019).
}

En suma, la llegada, presencia y participación de las 
mujeres en las Universidades e Instituciones de Educación Superior latinoamericanas y caribeñas, ha conllevado a un cierre de brechas y desigualdades que durante siglos fue la norma, pero también ha posibilitado un cambio al incorporar nuevos puntos de vista, metodologías y retos en pro de la construcción del país y la región.

Ahora bien, con este panorama expuesto, es posible continuar la reflexión retomando otro de los elementos esenciales en este texto: los orígenes y esencia de la Extensión Universitaria, de manera tal que, con ello presente, sea mucho más sencillo, analizar la actual presencia femenina en los procesos extensionistas latinoamericano a partir del estudio realizado por el autor del texto en tiempos recientes (2015-2020).

\section{Hitos de la extensión a la luz de la presencia femenina}

Aunque existen algunos antecedentes al acto fundacional de la Extensión Universitaria, es importante hacer claridad que a nivel mundial es considerado como hito, lugar y momento histórico en el que se reconoce el nacimiento de la llamada "Extensión universitaria" alrededor de 1867 en Cambridge, con los cuestionamientos del profesor y matemático James Stuart, un incansable defensor del acceso a la educación superior de las mujeres y de la clase trabajadora más vulnerable de la época, quien para ese entonces, se atrevió a retar la tradicional y estricta estructura de las universidades como claustros y templo académico, proponiendo a cambio una educación o estructura que cobijara a muchas más personas e involucrara en primera instancia al género femenino. Fue así como en (Cambridge) donde fue creado un sistema de conferencias extramuros implementado en las poblaciones aledañas que propiciaban el vínculo universidad-comunidad, con una mirada de lo que hoy podrían conocerse como los Cursos de Extensión Universitaria. Adicionalmente, Stuart estableció los apuntes de conferencias mejor conocidos como Syllabus, los que consistían, en su entonces, en un resumen de cada conferencia el que estaba diseñado para ayudar en primera instancia a las mujeres y demás estudiantes a seguir la charla, tomar apuntes y responder algunas preguntas. De esta manera, el profesor J. Stuart fue uno de los primeros en trasladar el aula de clases a las calles, parques o espacios no convencionales rompiendo los paradigmas de la época y pensando siempre en el acceso a la educación de las mujeres como parte de las luchas sociales por la igualdad de género (Valenzuela, 2021).

En 1869 siguiendo el ejemplo de dicha institución (Cambridge), Alemania a través de las universidades de Léipzig, Berlín, Hamburgo y Múnich, crearon una serie de cursos de enseñanza superior para todo público, que también incluían una serie de conferencias extramuros para las mujeres. Este modelo y dinámica continuó extendiéndose rápidamente a lugares como Manchester, Oxford, Liverpool, Sheffield, Leeds, el resto de Europa, y de manera subsiguiente, a los Estados Unidos (Princeton y Michigan):

"En 1900 la Universidad de Chicago, en Estados Unidos, crea la facultad de Extensión Universitaria. Un año después, la Universidad de Zaragoza, reestructura las conferencias de divulgación que venía desarrollando desde 1894 bajo el régimen de extensión universitaria. En 1902, también en España, en la Universidad de Valencia, se inauguran los cursos de Extensión Universitaria" (D’Andrea 2012)

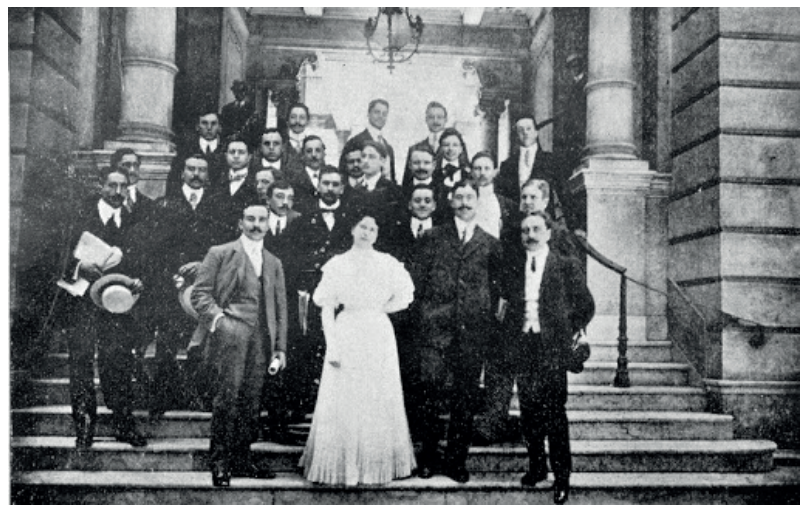

Primer Congreso Internacional de Estudiantes Americanos en 1908, en el centro Clotilde Luisi. Fuente: Fotografía tomada de Portal Universidad de la República: Eecuperado de: http://www.universidad.edu.uy/prensa/ renderltem/itemld/40140

En el caso del continente Americano, se destacan entre otros muchos acontecimientos: el Primer Congreso Internacional de Estudiantes Americanos celebrado en Montevideo en enero de 1908, en el que estuvieron presentes los reclamos de igualdad femeninos junto a la primera mujer abogada de américa latina (Clotilde Luisi) y más adelante, la Reforma de Córdoba (1918); escenario en el que se llevaron a cabo algunas disertaciones relacionadas

con la educación de la mujer y los problemas contemporáneos, permitiendo desde entonces que la Extensión fuera reconocida como una de las funciones sustantivas de las Universidades. En ambos casos, el 
reclamo y presencia de la mujer por su lucha al acceso a la educación son importantes en la consecución de acuerdos y nuevas metodologías que permitieran inclinarse por la extensión universitaria como medio de difusión del conocimiento y de la cultura en general.

El convencimiento, por la necesidad, de una universidad más cercana a las clases trabajadoras y femeninas se hizo cada vez más evidente, por lo que años más tarde, se lleva a cabo la "Primera conferencia latinoamericana de difusión cultural y extensión universitaria" organizada por la UDUAL(Chile,1957), donde se rescató la necesidad de permitir la participación de todos los actores (incluida la mujer) en el ejercicio y disfrute de la extensión y cultura universitaria. Asimismo, durante la Segunda Conferencia Latinoamericana de Extensión Universitaria y Difusión Cultural, realizada en México en 1972, el concepto es redefinido, como un proceso de interacción entre la universidad y los demás actores y componentes sociales del entorno. En agosto de 1991 nació la Asociación de Universidades del Grupo Montevideo o AUGM como respuesta de las nueve universidades con mayor desarrollo en políticas de investigación y extensión del Mercosur en defensa de la educación como un derecho de todos, indistintamente del género, raza, edad o filiación política. (Valenzuela, 2020).

Sumado a lo anterior, en el segundo semestre de 1993, de manera "informal" comenzaron los encuentros latinoamericanos de extensión universitaria, que posteriormente serían conocidos como CLEU (Congresos Latinoamericanos y caribeños de Extensión Universitaria) a cargo de la naciente Unión Latinoamericana de Extensión Universitaria mejor conocida como la ULEU. En 2008 con una gran delegación femenina y el respaldo de la Organización de Estados Iberoamericanos para la Educación, la Ciencia y la Cultura, surgió la declaración del Congreso Regional de Educación Superior CRES, realizado en la ciudad de Cartagena de Indias (Colombia), en la que se destacaba:

La educación superior como un bien público social, un derecho humano universal fundamental y un deber del Estado en el cual la Extensión Universitaria constituye una de las funciones sustantiva de las universidades $e$ instituciones de educación superior que traduce el compromiso con el desarrollo sustentable e integral de los países y el mejoramiento de la calidad de vida de los pueblos, bajo los principios de calidad, equidad, inclusión social, justicia, solidaridad, ética y compromiso social (CRES, 2008).

Hasta ese entonces $-\mathrm{y}$ dejando de mencionar muchos acontecimientos históricos-, los procesos extensionistas fueron liderados en su mayoría el género masculino quienes, no solo ocupaban altos cargos en las universidades, sino que concebían como destinatarios principales de la misma Extensión a las mujeres, niños y poblaciones vulnerables. Sin embargo, de manera reciente las universidades de la región, junto a diferentes Instituciones de Educación Superior, han venido incorporando a los equipos de las pro rectorías, vicerrectorías, direcciones, secretarias y jefaturas de extensión universitaria, proyección social, interacción o vinculación a un gran número de mujeres cambiando; así de manera notable la tradición original extensionista y permitiendo alcanzar casi la plena equidad de género en los roles jerárquicos o directivos de dicha función.

\section{Ser de la extensión universitaria}

A más de un siglo de la reforma educativa de Córdoba, el tema de la Extensión Universitaria como función misional continúa más vigente que nunca y cobrando gran relevancia en los procesos de formación y construcción de país-región, independientemente de la denominación que reciba en cada uno de los países latinoamericanos y caribeños, pues las universidades e instituciones de educación superior en general, poseen su propia mirada, definición e interpretación de la función sustantiva.

De allí que en cuanto al ser y quehacer mismo de la Extensión Universitaria sea mucho y a la vez poco lo que podría decirse, pues la Extensión es un proceso educativo no formal de doble vía, que se planifica de acuerdo a los intereses y necesidades de la sociedad. Este proceso ha evolucionado y ahora busca aprender y enseñar con las comunidades aportando a la toma de decisiones y formación de opinión; para generar conocimientos, al integrar la academia con el medio que la circunda, es una práctica que implica tanto equipos como procesos interdisciplinarios, es pensar

2Universidad Nacional de Asunción (Paraguay); Universidad de Buenos Aires, Universidad Nacional de La Plata, Universidad Nacional de Entre Ríos, Universidad Nacional de Litoral, Universidad Nacional de Rosario (Argentina); Universidad Federal de Santa María, Universidad Federal do Rio Grande do Sul (Brasil) y Universidad de la República (Uruguay)

3Constituida formalmente como institución no gubernamental sin fines de lucro el 21 de octubre de 1999. 
nuevos espacios y momentos fuera del aula que fortalezcan la formación integral desde un aprender haciendo. En otras palabras, la Extensión Universitaria es la cara amorosa y humanizadora de los procesos educativos desarrollados por las Instituciones de Educación Superior, con miras a una transformación social. Es aquella función que permite la integración de los diferentes actores universitarios (docentes, estudiantes, graduados, administrativos, no docentes y proveedores de las IES) de manera conjunta con los actores de la sociedad (Valenzuela, 2020).

Aludiendo a la Red Nacional de Extensión Universitaria de ASCUN, la extensión permite:

Establecer un diálogo permanente, respetuoso, riguroso y crítico entre los saberes especializados de la academia (científicos, tecnológicos y artísticos) y los saberes y experiencias sociales, posibilitando una integración activa entre las IES y las instituciones sociales, políticas, económicas y culturales. La extensión facilita la generación de espacios colectivos, como expresión del fortalecimiento de lo público en la sociedad (Red Nacional de Extensión Universitaria ASCUN, 2018)

Esta función constituye una de las actividades fundamentales del quehacer de la universidad y permite el engranaje armónico de las tres funciones misionales, como proceso horizontal e interdisciplinario ha de contribuir a orientar la investigación y la enseñanza, debe leerse en virtud del contexto del que forma parte, y como diría Humberto Tommasino:

Es un proceso vinculante, formativo y transformador". La extensión entonces, no solo es relevante dentro de la academia por los motivos arriba expuestos, sino que adicionalmente, les ofrece a las universidades la posibilidad de entender críticamente sus propias prácticas, repensarse institucionalmente y repensar sus políticas corporativas (Valenzuela, 2018).

Formas de realización de la extensión: Como bien se pudo apreciar en el acápite anterior, en sus inicios la Extensión Universitaria tenía como destinatarios principales a las mujeres y se desarrolló a través de cursos de extensión principalmente. Pese a ello, con el pasar de los años y la inclusión de las mujeres en los cargos directivos, roles jerárquicos y gestores extensionistas; la función sustantiva fue creciendo en estrategias, dinámicas, programas y metodologías, hoy conocidas como modalidades, tipologías, ámbitos de actuación o formas de realización.

Para poder entender un poco mejor el fenómeno reciente de la feminización de los espacios extensionistas, se hace necesario tener claridad de la manera cómo las universidades e instituciones de educación superior latinoamericanas y caribeñas gestionan actualmente dicha función sustantiva. Así las cosas, en términos generales y haciendo alusión a Patricio Donoso, es posible hablar de Extensión, a la luz de tres grandes ejes: extensión académica, extensión artístico-cultural y servicios de extensión. Desde allí, bien se podría decir que la extensión universitaria hoy en día se realiza entonces mediante 11 grandes formas a saber (ver anexo):

\section{- Educación continua o continuada}

- Prácticas y pasantías universitarias o Ejercicio Profesional Supervisado

- Servicios docentes asistenciales

- Prácticas solidarias, prácticas socioeducativas, aprendizaje servicio $\mathrm{o}$ proyectos sociales estudiantiles

- Relacionamiento con los egresados-graduados

- Gestión Cultural

- Voluntariado

- Servicios de asesorías y consultorías

- Interdisciplinarios de extensión o programas institucionales de servicio a la comunidad que integran formación e investigación

- Gestión de la Innovación o transferencia tecnológica o Gestión del emprendimiento

\section{- Proyectos comunitarios}

No cabe duda que con esta amplia gama de estrategias, modalidades o tipologías con las que se cuenta en la actualidad, el trabajo desarrollado con mujeres tanto en su rol de destinatarias como el de gestoras, líderes y directivas de la misma función extensionista, ha permitido el crecimiento exponencial de carácter muy 
diferente al de 1867, cuando nació la extensión en Cambridge.

\section{Discusión y resultados: mujeres líderes gestoras de la extensión universitaria}

Como pudo observarse con los argumentos planteados a lo largo de este texto, durante los primeros siglos de historia Extensionista, la participación de las mujeres como directivas, líderes o gestoras de la función sustantiva, fue en extremo limitada, por no decir que casi nula. Sin embargo, luego de la Reforma Universitaria de 1918, de manera paulatina, pero constante, se fue dando un quiebre en dicho esquema, permitiendo de a poco, que éstas asumieran cargos y roles directivos o de liderazgo de la función misional al interior de las universidades, en especial en la región latinoamericana y caribeña, lo que ha traído consigo una serie de cambios significativos sumado a la posibilidad de una universidad más solidaria y comprometida con un proyecto regional-global.

Las reflexiones anteriores y la fuerte presencia de las mujeres en la función de extensión, vinculación o proyección social durante los últimos cinco (5) años, llevaron al autor de este texto a abordar el tema de la feminización de los espacio extensionistas latinoamericanos y caribeños como objeto de estudio, realizando para ello un seguimiento aleatorio del rol femenino en las estructuras jerárquicas universitarias nacionales e internacionales ${ }^{4}$, junto a una revisión documental de la conformación de los equipos gestores y del ejercicio mismo, así como de la gestión y liderazgo de dicha función universitaria en último quinquenio; sumado a una serie de entrevistas realizadas a los responsables de la función universitaria en 20 países ${ }^{5}$ latinoamericanos y caribeños incluyendo 180 instituciones de educación superior consultadas (71 en Centroamérica y el Caribe, y 109 en Suramérica).

El estudio de carácter cuantitativo-descriptivo, contabilizó entonces la cantidad de mujeres y hombres involucrados en los equipos extensionistas, a partir de la información consignada en las diferentes páginas web institucionales, en lo que concierne a las 11 formas de hacer extensión ya mencionadas anteriormente. obtenidos con las entrevistas, a los responsables de la función en los 20 países de estudio, de manera tal, que fue posible determinar, a partir de ello el porcentaje femenino en los roles jerárquicos de dirección y liderazgo de la función extensionista en cada institución y país; acto seguido, se revisó el porcentaje de hombres y mujeres que integran las pro rectorías, vicerrectorías, direcciones, secretarías y jefaturas de extensión o proyección social en la actualidad, evidenciando entre otros lo siguiente:

a) La cantidad de hombres y mujeres que hacen parte de la extensión latinoamericana y caribeña, confirma el escenario reciente de feminización de la extensión universitaria (figura 1) enunciado por IESLAC en 2005.

b) La cantidad de hombres vs mujeres que se encuentran ejerciendo los roles de pro rectores(as), vicerrectores(as), directores(as), secretarios(as) o jefes de extensión universitaria en estos 20 países latinoamericanos, refleja la casi equidad de género, es decir, que el $51 \%$ del grupo es femenino, mientras que el $49 \%$ restante es masculino como indica la figura 2 avanzando de forma evidente en el cierre de brechas de desigualdad de género en la educación superior.

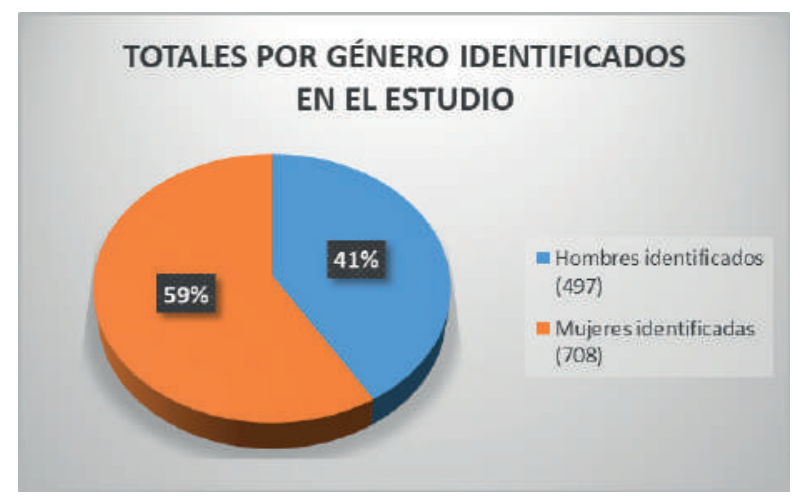

Figura 1. Distribución por género de la presencia en extensión universitaria a nivel latinoamericano y caribeño.

A partir de allí, se agregaron los datos estadísticos

4 Total de países consultados: 10 centroamericanos y 10 suramericanos.

5 Argentina, Bolivia, Brasil, Colombia, Costa Rica, Cuba, Chile, Ecuador, El Salvador, Guatemala, Honduras, México, Nicaragua, Panamá, Paraguay, Perú, República Dominicana, Puerto Rico, Uruguay y Venezuela. 


\section{DISTRIBUCIÓN DE ROLES JERÁRQUICOS DE LA FUNCIÓN DE EXTENSIÓN}

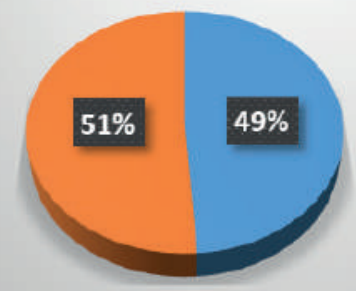

Figura 2. Porcentaje directivo-jerárquico latinoamericano de la función de extensión universitaria

c) El porcentaje de participación de acuerdo al género en los equipos de extensión universitaria, proyección social o vinculación de los 20 países analizados corresponde a un $60 \%$ vs $40 \%$, es decir, que, de un total de 1033 personas identificadas en el estudio a cargo de los procesos extensionistas, 417 eran hombres y 616 mujeres, tal como se puede apreciar en la figura 3 , proporción que se mantiene en el continente suramericano, pero que varía ligeramente en el caso de Centroamérica y el Caribe (Figuras 4 y 5 ).

d) Ahora, con relación al género mayoritario que desempeña cargos jerárquicos o directivos de extensión universitaria en lasinstituciones de educación superior, fue posible evidenciar que para el caso de Centroamérica, el género masculino se encuentra al frente con un $52 \%$, mientras que en Suramérica es el género femenino el que ocupa estos roles jerárquicos con el $53 \%$ (figura 6). Sin embargo, en cuanto a la manera cómo están conformados los equipos de gestión de esta función universitaria, se pudo observar que en Centroamérica el $41 \%$ es masculino vs el $69 \%$ femenino; entre tanto en Suramérica, el $40 \%$ es masculino vs $60 \%$ femenino (figura 7). Lo anterior permite evidenciar que dichos datos y análisis, se encuentran en línea de las investigaciones realizadas y citadas en este texto, por algunas universidades de la región durante la última década.

\section{CONFORMACIÓN LATINOAMERICANA DE LOS EQUIPOS DE EXTENSIÓN UNIVERSITARIA}

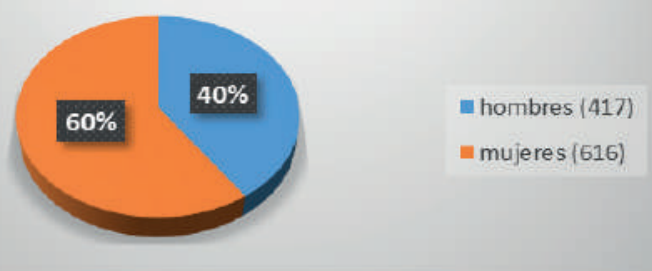

Figura 3. Porcentaje de conformación según el género de los equipos extensionistas en los 20 países consultados

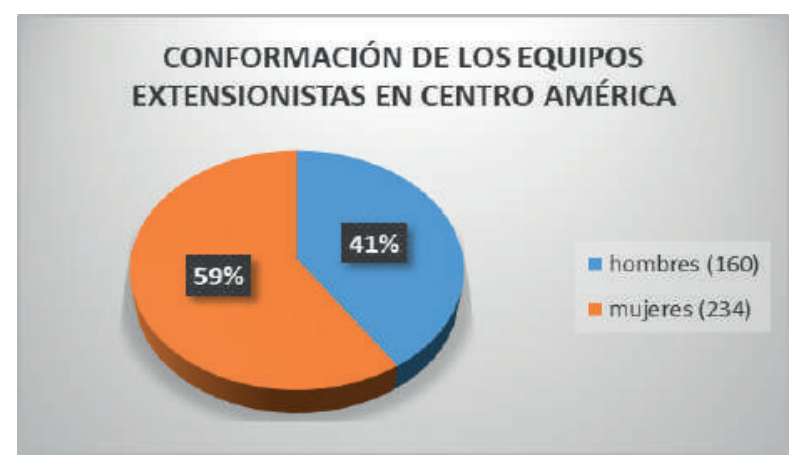

Figura 4. Conformación centroamericana y caribeña de los equipos extensionistas.

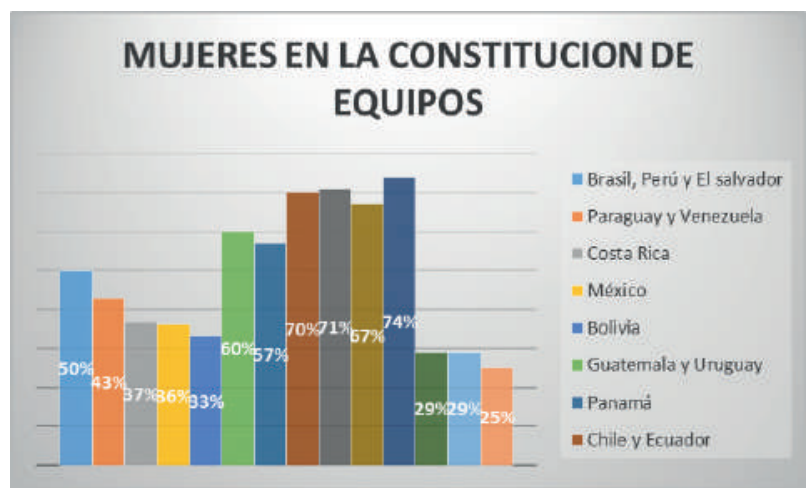

Figura 5. Porcentaje de mujeres en la constitución de los equipos según cada país consultado. 


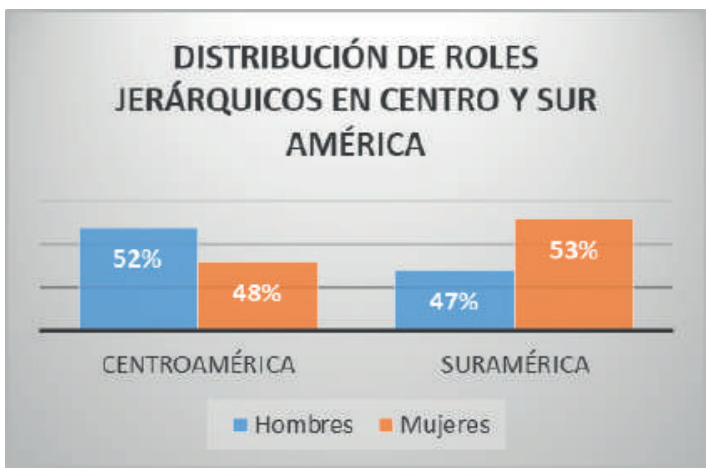

Figura 6. Relación de los cargos directivos o jerárquicos de la función de extensión en centro y Suramérica.

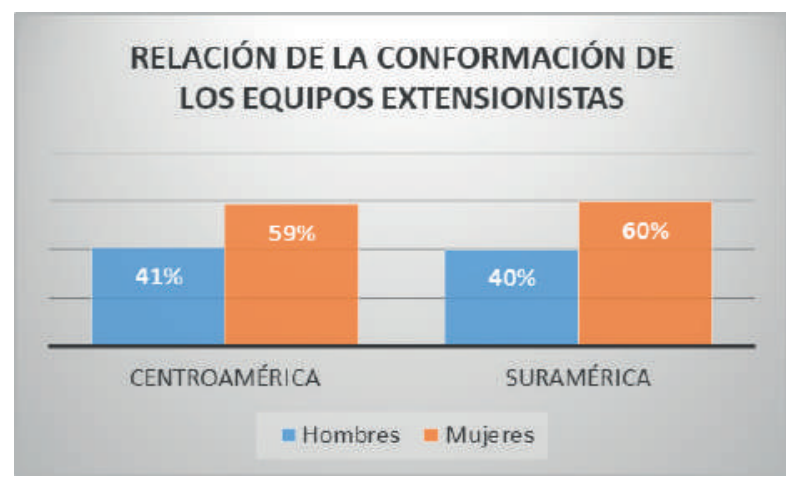

Figura 7. Relación de la conformación de los equipos extensionistas en centro y Suramérica.

e) Ocho (8) de los 20 países consultados en este estudio, contaban con mujeres al frente de los cargos directivos de la extensión (figura 8); solo tres (3) presentaban igualdad de género y los nueve (9) restantes dejaban estos roles para los hombres (figura 9). Es decir, que aún existe un camino por recorrer,

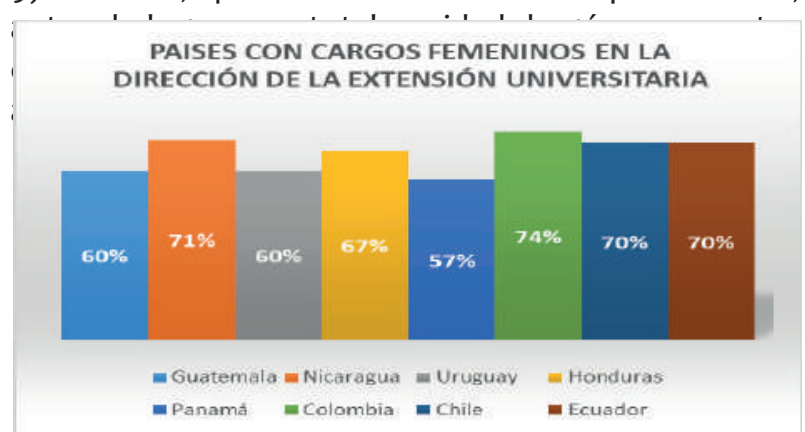

Figura 8. Porcentaje de mujeres en los cargos directivos o jerárquicos de la función de extensión en centro y Suramérica.

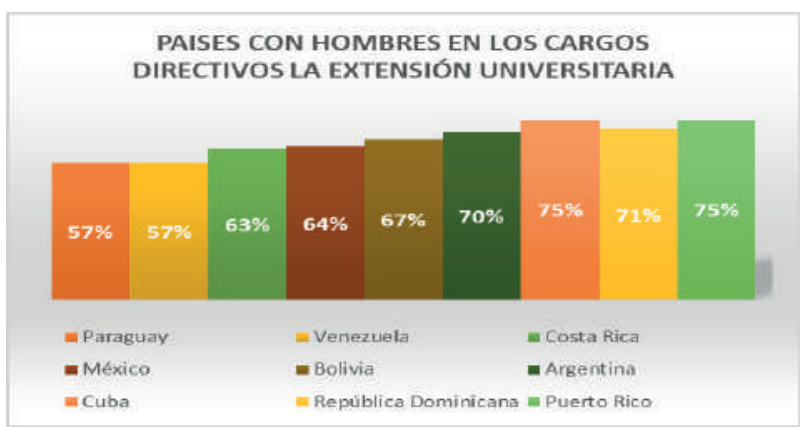

Figura 9. Porcentaje masculino en los cargos directivos de la Extensión.

Ahora bien, esta misma información fue revisada y analizada de manera particular para cada uno de los países incluidos en este estudio y llamó la atención del autor de este texto, las cifras en cada uno de los casos particulares de estos 20 países analizados, dado que en algunos de ellos la información variaba bastante, pese a ser contextos aparentemente similares, mientras que, en otros, se encontraban panoramas muy parecidos en contextos o ubicaciones geográficas distantes. Para poder entender un poco mejor esta afirmación a continuación, se presenta de manera más detallada algunos hallazgos:

Roles jerárquicos o cargos directivos:

- Tres países de la región, han avanzado en el cierre de brechas de desigualdad de género, en los espacios extensionistas universitarios. Lo anterior está basado en los resultados del estudio con relación a los casos de Brasil, Perú y el Salvador, países que presentan igualdad de género en los cargos directivos de la función universitaria de Extensión o Proyección social, sin embargo, es necesario, como región latinoamericana, continuar trabajando en pro de esta igualdad de género, pues existen aún lugares muy lejanos a alcanzarla.

- Casos como los de Paraguay y Venezuela, dejan entrever un caminar un poco más cercano a la anhelada igualdad de género en el ámbito extensionista pues presentan una pequeña diferencia a favor del género masculino (57\%) que quizás obedezca a los años de lucha y logros alcanzados en el siglo pasado. Por otro lado, en la región existen otros países como Guatemala y Uruguay que inclinaron la balanza en el 
sentido contrario, es decir, que han permeado su estructura jerárquica administrativa de la extensión o proyección social con un avance feminista evidenciado en un $60 \%$ la preferencia de mujeres en los roles jerárquicos-directivos de la extensión universitaria.

- Esta feminización de los roles directivos de la función universitaria al interior de las diferentes instituciones de educación superior, sigue siendo una constante en crecimiento en países como Chile, Ecuador, Nicaragua, Honduras y Colombia donde se prefiere en estos roles jerárquicos al género femenino con un 67 a $74 \%$, mientras que en otros países con contextos un poco similares (Costa Rica, México y Bolivia) el camino por recorrer sigue estando pendiente debido a que se ha optado por dejar en dichos cargos al género masculino con un $64 \%$.

Quizás los países que presentan al momento un camino largo y pendiente de recorrer al respecto de este tema, son Puerto Rico y Cuba con un $75 \%$ de hombres en el ejercicio de la dirección institucional de la función, seguidos de Argentina y República Dominicana que, a pesar de tener en sus equipos una cantidad notable de mujeres, aún persisten con el $70 \%$ masculino en estos roles jerárquicos.

Conformación de equipos extensionistas (figura 10):

- Los resultados del estudio han demostrado que en el momento de constituir los equipos gestores y organizativos de la extensión, vinculación o proyección social, las universidades latinoamericanas confían a las mujeres dicha responsabilidad. Afirmación que se respalda en casos como los de Ecuador, Nicaragua, Honduras, Costa Rica, México, Panamá, Perú, Chile, Venezuela, Colombia y Bolivia que presentan un $67,3 \%$ en promedio de mujeres en los equipos.

- Con respecto a Latinoamérica y el Caribe cuenta con grandes ejemplos de equidad en este punto. Argentina, Paraguay, El Salvador, Uruguay y Guatemala han logrado conformar equipos gestores mixtos en un $50,6 \%$ en promedio con grandes resultados.

- Los desafíos para las instituciones universitarias independientemente de su carácter público o privado en países como República Dominicana,

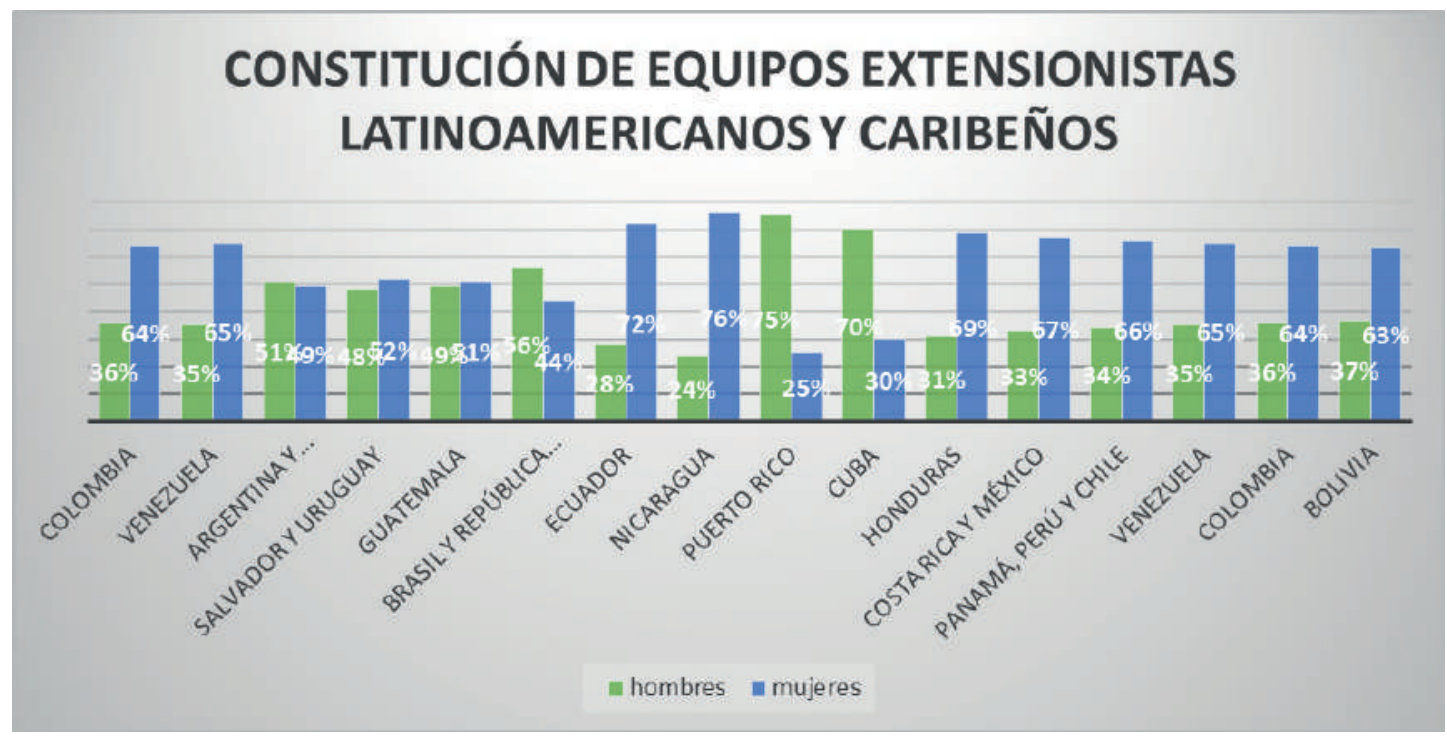

Figura 10. Constitución de equipos extensionistas latinoamericanos. 
Brasil, Puerto Rico y Cuba siguen trabajando por un cierre de brechas de género en sus equipos extensionistas pues cuentan en promedio con una mayoría masculina en sus equipos de $64,2 \%$.

\section{Análisis individual por país:}

- En el caso de Costa Rica aparentemente quienes dirigen la función de extensión son las mujeres con un $63 \%$, lo mismo sucede con la conformación de sus equipos en un $67 \%$.

- Cuba por su parte, cuenta con una dirección extensionista jerárquica masculina representada en el $75 \%$. Los equipos vinculados a la Extensión bajo las diferentes tipologías de la Extensión Universitaria, se dividen en un $70 \%$ hombres vs $30 \%$ mujeres.

- En El Salvador la función sustantiva la dirigen de manera igualitaria hombres y mujeres y los equipos se encuentran conformados en un $52 \%$ por mujeres y $48 \%$ hombres.

- En el caso de Guatemala, la información recolectada muestra que son las mujeres con un $60 \%$ quienes lideran o dirigen la función universitaria y que los equipos involucrados desde las universidades en la planeación y ejecución de la extensión están conformados por un $51 \%$ mujeres y $49 \%$ hombres.

- Para el caso de Honduras las universidades consultadas evidencian un rol directivo de preferencia femenino del $67 \%$, con una conformación de equipos del $69 \%$ femenino y $31 \%$ masculino.

- En México sucede lo contrario, pues la función universitaria pareciera estar dirigida en un $64 \%$ por el género masculino y contar con un $67 \%$ de mujeres en la conformación de equipos vs un 33\% de hombres.

- En Nicaragua son las mujeres con el $71 \%$ quienes lideran o ejercen roles jerárquicos directivos de extensión universitaria y los equipos institucionales extensionistas se encuentran conformados en un $76 \%$ por mujeres y un $24 \%$ por hombres.

- Panamá se suma a los países en los que son las mujeres quienes dirigen la Extensión universitaria, en este caso con un $57 \%$ y cuentan con equipos conformados en un $66 \%$ por mujeres vs un $34 \%$ por hombres.

- Puerto Rico cuenta con un liderazgo directivo masculino del $75 \%$ en la función sustantiva, lo que concuerda con una conformación también mayoritaria masculina de los equipos en igual porcentaje (75\% hombres).

- En cuanto a República Dominicana, ésta se suma a la lista de países con liderazgo o dirección masculina de la extensión con el 71\% de hombres al frente las vicerrectorías, direcciones, secretarias o jefaturas de extensión. Ahora, con en relación a la conformación de sus equipos según el género, éstos cuentan con un $56 \%$ masculino y un $44 \%$ femenino.

- El caso de Argentina es ligeramente similar al anterior, con un $70 \%$ hombres directivos y una conformación de equipos institucionales extensionistas de $51 \%$ hombres vs $49 \%$ mujeres,

- Al analizar la información en Bolivia, se encontró que también son los hombres quienes ejercen roles jerárquico directivos de la extensión con un $67 \%$ y que los quipos de la universidades e instituciones de edición superior a cargo de las diferentes modalidades o tipologías cuentan con un $63 \%$ del género femenino y un $37 \%$ del género masculino.

- En Brasil, al igual que en El Salvador y Perú, existe igualdad de género en el ejercicio mismo de las pro rectorías, vicerrectorías, direcciones, secretarías o jefaturas de extensión. Sin embargo, al momento de confirmar los equipos institucionales de dicha función universitaria, se opta por una mayoría masculina con el $56 \%$.

- Chile, por su parte, cuenta con un 70\% de cargos femeninos directivos en la función de extensión y equipos conformados en su mayoría por mujeres con un $66 \%$ vs $34 \%$ de hombres.

- El caso de Colombia es un poco diferente a todos los anteriores, pues es el país de la región analizado que presenta el mayor porcentaje de mujeres a cargo de esta función sustantiva (74\%), y por ende, la menor cifra de hombres en cargos directivos extensionistas (26\%), privilegiando de igual manera en la conformación de sus equipos al género femenino con un $64 \%$.

- En Ecuador, aparentemente quienes "lideran" 
o desempeña roles jerárquicos al interior de la instituciones de educación superior en torno a la función de extensión, son las mujeres con el 70\%, y casi en igual proporción la conformación de sus equipos ( $72 \%$ femeninos vs $28 \%$ masculinos).

- Paraguay cuenta con roles directivos extensionistas masculinos representados en el $57 \%$, lo mismo sucede al analizar la conformación institucional de sus equipos donde el $51 \%$ son hombres y el $49 \%$ mujeres.

- Al revisar el caso de Perú, la información recolectada mostró una equidad de género en la asignación de las vicerrectorías, direcciones, secretarias o jefaturas de extensión; no obstante, los equipos institucionales de estas dependencias se encuentran conformados en su mayoría por el género femenino con un $66 \%$.

- En el caso de Uruguay, son las mujeres quienes aparentemente ejercen cargos directivos de la función de extensión con una diferencia porcentual del $60 \%$, mientras que, en cuanto a la conformación de los equipos institucionales extensionistas, la diferencia se reduce a un $52 \%$ femenino vs $48 \%$ masculino.

- Finalmente, al revisar la información recopilada para el caso de Venezuela, los datos obtenidos evidenciaron un rol masculino que desde las pro rectorías, vicerrectorías, direcciones, secretarías o jefaturas de extensión "lidera" o dirige la función universitaria con el $57 \%$. Ahora, con relación a la manera como los equipos institucionales a cargo de alguna de las 11 tipologías de extensión se encuentran conformados, la información arrojó que el $35 \%$ sin hombres mientras que el $65 \%$ mujeres.

En suma, $8^{6}$ de los 20 países de estudio (40\%) tienen preferencia por el género femenino en los roles directivos de la Extensión, Proyección social, Vinculación con el medio, Interacción o Relación Universidad-Sociedad y 92 instituciones de educación superior — de las 180 consultadas en la regiónoptan por contratar o vincular en dichos cargos a las mujeres (figura 11). Aspecto similar que se presenta en el momento de revisar la manera como se

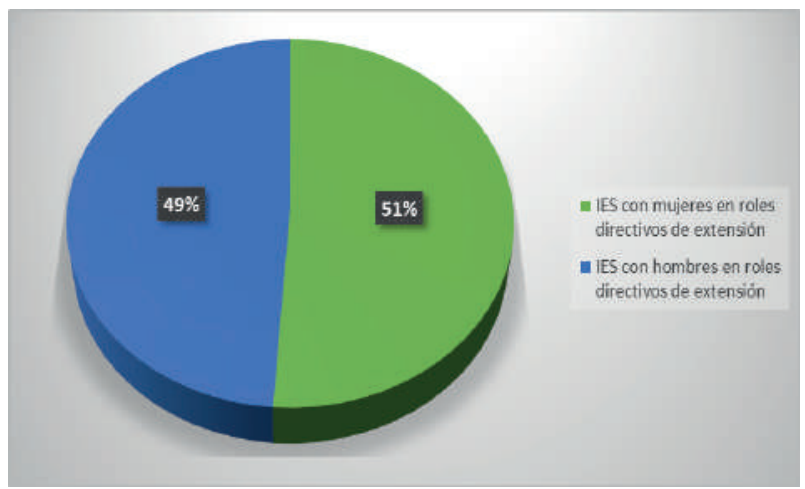

Figura 11. Porcentaje de Instituciones con roles femeninos y masculinos en los cargos directivos de la Extensión.

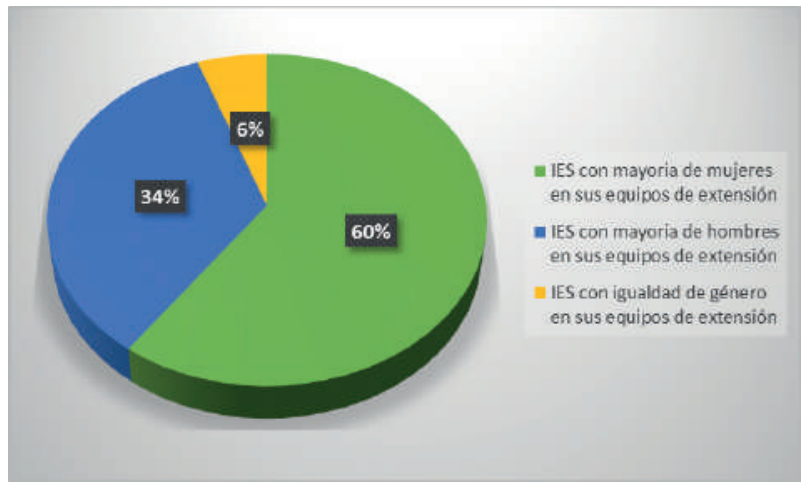

Figura 12. Porcentaje de Instituciones de Educación Superior según la conformación de los equipos de extensión.

encuentran conformados los equipos institucionales a cargo de la gestión e implementación de las diferentes modalidades o tipologías de la Extensión: 108 instituciones presentan un porcentaje mayor de mujeres en sus equipos, mientras que solo 10 tienen igualdad de género en los mismos. (Figura 12).

\section{Conclusiones}

La extensión universitaria, proyección social, vinculación con el medio, interacción o relación universidad-sociedad, —según como se denomine en los países latinoamericanos y caribeños-, ha crecido en estrategias y metodologías desde sus inicios en Cambridge en 1867, cambiando incluso la manera como se relaciona con la mujer: ya no como aquel destinatario de su ejercicio, sino, como el actor capaz de dirigir, liderar y gestionar exitosamente la función universitaria. En esa medida, muchas instituciones de educación superior pública y privada de América 
Latina y el Caribe, cuentan en la actualidad con equipos extensionistas femeninos en su mayoría, así como, con valiosas mujeres al frente de las pro rectorías, vicerrectorías, secretarías, direcciones y jefaturas de dicha función sustantiva.

La afirmación y predicción del Instituto Internacional para la Educación Superior en América Latina y el Caribe (IESALC) de la UNESCO realizada en el año 2005 en cuanto a que el proceso de feminización de la educación superior se presentaba para ese entonces como un hecho irreversible, creciente y que traería impactos sociales de transformación de las instituciones y centros de investigación latinoamericanos (Agostini, 2017). Hoy más de tres quinquenios después, es una total realidad evidenciada en las diferentes cifras y estudios de las universidades y organismos internacionales de la región. La presencia femenina en los entornos universitarios y en especial en los espacios extensionistas, se convierte en una gran oportunidad para desarrollar políticas y programas que aborden entre otros, las necesidades de las mujeres en torno a la educación superior, así como también reformas mucho más inclusivas con perspectiva de género, tal como lo afirma Stefania Giannini, Directora General Adjunta de la UNESCO en un reciente informe con motivo del día internacional de la mujer.

Los movimientos sociales y feministas regionales son un complemento y aliado de las instituciones de educación superior $y$, en consecuencia, de la misma extensión universitaria, vinculación o proyección social. Por lo tanto y parafraseando a Sandra Araya, es imperativo generar sinergias que permitan nutrir las tres funciones sustantivas de las universidades (investigación, la docencia y la extensión universitaria) transformando desde adentro los claustros universitarios y educativos con miras a que en un mediano y largo plazo, ello genere transformación de las condiciones sociales que propician la desigualdad y aqueja a la región.

Los cuestionamientos y teorías alrededor de este proceso de feminización de la extensión universitaria tienden a considerarlo como fruto de una mayor sensibilización femenina al detalle ligada a la capacidad de observar y ser creativos en el momento de solucionar problemas. Adicionalmente, las disertaciones ponen sobre la mesa del debate el que quizás este cambio y migración a la feminización de los espacios extensionistas latinoamericanos y caribeños, esté relacionado con los procesos de empatía respecto a las problemáticas sociales del día a día, alteridad y otredad femeninos; mientras otros opinan que es debido la capacidad femenina de persuasión, al potencial de éstas para desarrollar múltiples acciones de manera paralela, a su recursividad y practicidad a la hora de gestionar y liderar. Sin embargo, lo que si es cierto, es que la creciente y gran presencia femenina, tanto en la dirección administrativa como en la gestión de la extensión en las universidades e instituciones de educación superior latinoamericanas y caribeñas, puede estar relacionada con su capacidad de trabajo en equipo, sus niveles de resilencia, capacidad para reconocer los entornos y ejecutar o gestionar de manera asertiva y efectiva las tareas (no se puede olvidar que en un alto porcentaje, ésta mujeres son profesionales exitosas, madres, esposas, compañeras y amigas) sin que con ello se quiera decir que el género masculino no lo puede hacer.

La discusión por la feminización de la extensión ha impulsado nuevos debates, estrategias y relaciones con los diferentes actores universitarios, tanto al interior como al exterior de los campus, lo que implica un trabajo de equidad, interdisciplinariedad y transformación que puede ser objeto de reflexión desde múltiples miradas iniciando por la concepción maternal y la capacidad de las mujeres para socializar con las comunidades o territorios de una manera más pronta y cercana.

Finalmente, no cabe duda de que la presencia femenina -en lo referente a la dirección-liderazgo y gestión de la extensión universitaria - es una puesta por la proyección de un agente inspirador y transformador, aportando a la construcción de tejido social, país y región.

\section{Referencias bibliográficas}

- Agostini, A. y Schiavi, N. (2017). Informe la presencia de las mujeres en la universidad y su participación Política en órganos de gestión y representación. Observatorio de Derechos Humanos de la Universidad Nacional de Córdoba

- Araya Umaña, S. (2014). Políticas de igualdad de género y educación superior: desafíos conceptuales y prácticos. En: Seminario Internacional calidad de la educación superior y género en américa latina. FLCSO. Quito 
- Azuaje Rondón, V. (2014). Mujeres en la Educación Superior. Una mirada desde Venezuela. Integración $\mathrm{Y}$ Conocimiento, 3. Recuperado a partir de https://revistas.unc.edu.ar/index.php/ integracionyconocimiento/article/view/9250

- Corzo Morales, M., \& Galeano Camacho, E. (2020). Las mujeres ante la educación superior en Colombia: una aproximación general. Foro por la vida, 3(10), 83104.

- CRES. (2008). Declaración de la Conferencia Regional de Educación Superior en América Latina y el Caribe. Cartagena, Colombia: UNESCO- IESLAC

- D’Andrea, R. E, Zubiría, A y Sastre Vázquez, (2012). Reseña histórica de la extensión universitaria. Secretaria de la Universidad Nacional del Centro de la Provincia de Buenos Aires P1-12

- Dirección de proyección social Universidad de San Buenaventura (USB), sede Bogotá (2016). Programa de relacionamiento con egresados. Bogotá.

- Donoso P. (2001). Breve historia y sentido de la extensión universitaria. Revista Calidad en la Educación. 15, 177-188 Santiago de Chile: Consejo Nacional de Educación (CNED)

- Matteucci, F. (2014). Women in Italian astronomy. Universita di Trieste, Chair of INAF Scientific Council, Osservatorio Astronomico di Padova. Recuperado de https://arxiv.org/ftp/arxiv/papers/1402/1402.1952. pdf

- Moraima Romero, D., \& Aldana Zavala, J. (2019). Proyectos Comunitarios en el proceso de Transformación Social de la Universidad. CIENCIAMATRIA, 5(9), 151 - 167. https://doi. org/10.35381/cm.v5ig.104

- FLACSO (1991). Mujeres latinoamericanas en cifras, 1991

- Observatoriode Derechos Humanos delaUniversidad Nacional de Córdoba. (2017). La presencia de las mujeres en la Universidad y su participación política en órganos de gestión y representación.

- Organización de las Naciones Unidas para la Educación, la Ciencia y la Cultura UNESCO. (2021). Women in higher education: has the female advantage put an end to gender inequalities? París, Francia.
- Palermo, Alicia Itatí (2006). El acceso de las mujeres a la educación universitaria. Revista Argentina de Sociología, vol 4 No (7),11-46. ISSN: 1667-9261. Disponible en: https://www.redalyc.org/articulo. oa?id=26940702

- papadópulos, J. y RAdAKOVICH, R. (2003). Educación Superior y género en Uruguay. En Primer Seminario Internacional Los Géneros en la Educación Superior en América Latina y el Caribe, México, DF.

- Red Nacional de Extensión Universitaria. (2018). Política de Extensión: por una universidad comprometida con el país. ASCUN. Bogotá, Colombia.

- Rojas Herrera S. Feminización de la Extensión costarricense desde una perspectiva intergeneracional. En: Lovo S.M; Ramos M y Valenzuela S.M. (2019). Diálogos de saberes y conocimientos con compromiso social. Editorial Bonaventuriana.

- Secretaría de Cultura y Extensión Universitaria de la UNLPam (2020). "Mujeres y extensión universitaria. Un proceso transformador” http://contexto.unlpam. edu.ar/index.php/articulos/vinculacion/43-mujeres-yextension-universitaria-un-proceso-transformador.

- Rojas, J. y López C. (2015). Glosario de términos que se Utilizan en extensión Universitaria. Puyo, Ecuador. Unión Latinoamericana de Extensión Universitaria ULEU y Universidad Estatal Amazónica UEA.

- Valpuesta Fernández, M. (2012). Mujer y Universidad. Revista Historia De La Educación Latinoamericana, (4). Recuperado a partir de https://revistas.uptc.edu. co/index.php/historia_educacion_latinamerican/ article/view/1463

- ValenzuelaTovar, S.M.(2018).Unión Latinoamericana de Extensión Universitaria: un espacio de cooperación, intercambio y reflexión en materia de extensión y vinculación con la sociedad. $+\mathrm{E}$ : Revista de Extensión Universitaria, 8 (8), enero-junio, 61-88. doi: 10.14409/extensión.v8i8.Ene-Jun.7720.

- Valenzuela Tovar S.M. (2020). Evolución histórica de la extensión universitaria. Universidad de San Carlos de Guatemala USAC. Guatemala.

- Valenzuela Tovar S.M. (2020). La Extensión universitaria, una mirada desde la Cultura y la Danza. En: Primer encuentro danzario arte y academia. Universidad Distrital Francisco José de Caldas. 
Bogotá.

\begin{tabular}{c} 
Nicaragua, Managua. \\
Anexo \\
Educación Continua o \\
continuada \\
\hline \\
Prácticas y pasantías \\
universitarias o ejercicio \\
profesional supervisado
\end{tabular}

\section{Extensión Académica}

cional Autonoma de

Cursos, seminarios, especialidades (sin grado académico) o diplomados sobre temas específicos, enfocados a la mejora continua de los egresados y/o profesionales de diversas ramas. *Conjunto de procesos pedagógicos realizados con el propósito de formar, capacitar, actualizar, entrenar, acreditar y calificar grupos de personas en temas relacionados con necesidades sentidas de formación corta, intereses particulares de grupos, empresas, entidades, las ONG e instituciones en un área de conocimiento, o en la solución de problemas específicos. (Rojas, 2015).

Espacio de aproximación a escenarios laborales reales mediante la adquisición o aplicación de los conocimientos, valores y competencias que han adquirido los estudiantes durante el programa académico que cursan, en el campo de las actividades relacionadas con éste. Las asignaturas prácticas constituyen el espacio de aprendizaje elegido por programa académico para asegurar la adquisición de competencias profesionales a través de la aproximación a un escenario real de trabajo. En este sentido, la integración curricular se hace evidente a través de la evaluación del aprendizaje, en donde el rol de profesor cobra relevancia en la medida que apoya el proceso de construcción y uso creativo del conocimiento para apoyar la toma de decisiones frente a problemas reales. Por su parte la pasantía "es una opción de grado para optar por el título profesional, a través de la cual los estudiantes demuestran en escenarios laborales reales los conocimientos, habilidades, actitudes y valores adquiridos dentro su proceso formativo. (ASCUN, 2018).

Desarrollo de un modelo académico de servicio social y a la comunidad, desarrollado por los docentes, según el área de conocimiento o campo del

Servicios docentes asistenciales saber en el que se desenvuelve su labor y de acuerdo con la reglamentación curricular y administrativa-institucional. En los servicios docentes pueden estar incorporados estudiantes como parte de su formación. Pueden considerarse en esta categoría los consultorios jurídicos, psicológicos y unidades asesoras de libre concurrencia para usuarios externos. (Rojas, 2015)

Son consideradas como procesos educativos que se desarrollan fuera del espacio formal, estrategias institucionales de las universidades para la enseñanza y aprendizaje de los estudiantes a partir de la inserción en las comunidades con contextos y problemáticas reales.

Prácticas solidarias, socioeducativas, aprendizaje servicio o proyectos sociales estudiantiles
Incluyen en su desarrollo espacios de sensibilización, conceptualización, problematización, reflexión, intervención comunitaria específica y sistematización sobre lo vivido.

Estas prácticas no reemplazan las prácticas pre-profesionales ni las pasantías como actividad obligatoria para optar por la titulación y se convierten en una oportunidad para la innovación en educación. 


\section{Extensión artístico-culbural}

\section{Gestión cultural}

\section{Relacionamiento con los egresados-graduados}

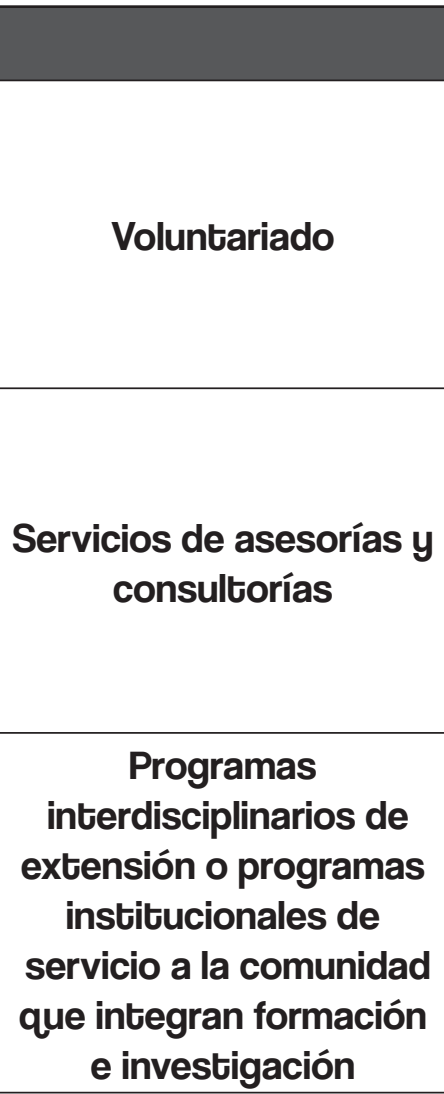

El arte o técnica de planificar y organizar, dirigir y liderar, supervisar y administrar las actividades propias del sector cultural en un marco organizativo determinado; en consecuencia, puede decirse que la gestión cultural universitaria es el arte de planificar y organizar, liderar y dirigir, supervisar y administrar aquellas actividades mediante las que la Universidad realiza su tercera misión. Entre ellas se incluyen la programación de la oferta y su financiación, la dirección de estructuras de gestión o servicios específicos, el mantenimiento de las infraestructuras correspondientes y la promoción de la participación cultural tanto interna como externa. (Ariño, 2015)

Implementa estrategias y proyectos, a partir de una propuesta operativa de comunicación, participación, formación y desarrollo humano, que delimita las áreas de acción y acompañamiento, como lineamientos institucionales acordes a las necesidades de la relación egresado? Universidad. Para ello, es necesario a) el manejo de una relación dialógica a través de medios amigables y efectivos para el acercamiento, seguimiento y apoyo; b) la motivación e inclusión de los egresados como agentes activos en los procesos institucionales en corresponsabilidad o acción de pertenencia, y por último c) propiciar espacios institucionales que favorezcan el crecimiento continuo de los egresados y el desarrollo de las dimensiones que los constituyen como personas. (USB, 2016).

\section{Servicios de Extensión}

Las redes y grupos de voluntarios universitarios se configuran en una de las expresiones de la responsabilidad social universitaria y la extensión solidaria. Su quehacer debe estar orientado al mejoramiento de la calidad de vida de las poblaciones menos favorecidas, vulnerables o vulneradas, a través de procesos participativos y de generación de capacidades en ellas, trascendiendo de ese modo las prácticas asistencialistas. (ASCUN, 2018).

Acciones orientadas a resolver demandas y necesidades específicas de los diferentes agentes sociales y comunitarios, buscando encontrar, a nivel técnico, económico o social, las soluciones más adecuadas a las problemáticas existentes. Este apartado comprende las acciones que posibilitan la transferencia de ciencia y tecnología, viables y útiles para enfrentar situaciones concretas y problemáticas existentes en el medio. También caben aquí los servicios relacionados con la identificación e investigación de problemas y la sustentación de propuestas viables para la solución de estos. (ASCUN, 2018).

De caracteres inter y transdisciplinario, que se desarrollan alrededor de un campo de acción o sector específico a largo plazo, relacionados con las políticas institucionales y con las problemáticas de las comunidades en los ámbitos nacional, regional y local. Articulan simultáneamente la formación y la investigación con la extensión, vinculan a profesores y estudiantes, y se desarrollan mediante procesos sustentables de gestión prospectiva y estratégica. Estas actividades deberán contar con el apoyo de la institución educativa para garantizar su funcionamiento permanente (ASCUN, 2018). 


\begin{tabular}{|c|c|}
\hline \multicolumn{2}{|r|}{ Servicios de Extensión } \\
\hline Proyectos comunitarios & $\begin{array}{l}\text { Todo aquel que tenga como objetivo promover la cultura en cualquiera de } \\
\text { sus expresiones, (científica, económica, jurídica, política, artística, literaria, } \\
\text { deportiva, etc). } \\
\text { Son concebidos como instrumentos en los cuales se plantea la solución a } \\
\text { un problema o la satisfacción de una necesidad sentida por la colectividad; } \\
\text { es decir, son planes de acción detallados que resuelven un problema, } \\
\text { una necesidad colectiva, situacional, corresponsables y cogestionaria de } \\
\text { la comunidad, de esta manera, a través de los proyectos comunitarios se } \\
\text { puede avanzar en la construcción de una mejor sociedad; democrática, } \\
\text { participativa y protagónica, que consolide valores de bien común, una sana } \\
\text { y productiva convivencia. (Moraima Romero, D 2019). }\end{array}$ \\
\hline $\begin{array}{l}\text { Gestión de la Innovación } \\
\text { o transferencia } \\
\text { tecnológica o Gestión del } \\
\text { emprendimiento }\end{array}$ & $\begin{array}{l}\text { Se expresa a través de la generación e incubación de empresas spin-off y } \\
\text { start up, implementadas a través de diferentes modelos que flexibilizan y } \\
\text { facilitan la puesta en marcha de este tipo de empresas. Estas, contribuyen a } \\
\text { diversificar la industria nacional y a desarrollar vocaciones económicas con } \\
\text { mayor valor agregado, para el caso de los emprendimientos empresariales } \\
\text { de base tecnológica; a potenciar las expresiones y manifestaciones } \\
\text { culturales, para el caso de los emprendimientos culturales; y a atender } \\
\text { necesidades de la población, cuando se trata de emprendimientos sociales... } \\
\text { La Gestión de la Innovación incluye de manera adicional lo relacionados } \\
\text { con los Parques tecnológicos como un instrumento fundamental para la } \\
\text { incubación de empresas tecnológicas... (ASCUN, 2018). }\end{array}$ \\
\hline $\begin{array}{l}\text { Programas } \\
\text { interdisciplinarios de } \\
\text { extensión o programas } \\
\text { institucionales de } \\
\text { servicio a la comunidad } \\
\text { que integran formación e } \\
\text { investigación }\end{array}$ & $\begin{array}{l}\text { De caracteres inter y transdisciplinario, que se desarrollan alrededor de un } \\
\text { campo de acción o sector específico a largo plazo, relacionados con las } \\
\text { políticas institucionales y con las problemáticas de las comunidades en los } \\
\text { ámbitos nacional, regional y local. Articulan simultáneamente la formación } \\
\text { y la investigación con la extensión, vinculan a profesores y estudiantes, y } \\
\text { se desarrollan mediante procesos sustentables de gestión prospectiva y } \\
\text { estratégica. Estas actividades deberán contar con el apoyo de la institución } \\
\text { educativa para garantizar su funcionamiento permanente (ASCUN, 2018). }\end{array}$ \\
\hline
\end{tabular}

